\title{
A study of $2175 \AA$ absorption feature with TAUVEX: An Indo-Israeli UV mission
}

\author{
C. Muthumariappan ${ }^{1}$, G. Maheswar ${ }^{2,3}$, C. Eswaraiah ${ }^{3}$, and \\ A. K. Pandey ${ }^{3}$ \\ ${ }^{1}$ Vainu Bappu Observatory, Indian Institute of Astrophysics, Kavalur India \\ email: muthu@iiap.res.in \\ ${ }^{2}$ Korea Astronomy \& Space Science Institute, Daejeon, R. O. Korea \\ email: maheswar@kasi.re.kr \\ ${ }^{3}$ ARIES, Nainital, India \\ email: eswarbramha@aries.ernet.in, pandey@aries.ernet.in
}

\begin{abstract}
TAUVEX is an Indo-Israeli collaborative mission to make photometric observations in the UV region. Using the narrowband filters positioned near $2175 \AA$ feature, we plan to construct the UV bump in the extinction curve towards B type stars brighter than $\mathrm{m}_{v}=14$. Archival data of TD1, Galex missions are used for stars brighter than $\mathrm{m}_{v}=10$. We sample the distance to obtain the extintion at different location of the local ISM upto $2 \mathrm{kpc}$. Using a dust model having silicate, graphite and $\mathrm{PAH}$ as components, the extinction curve at different location of the Galaxy is fitted to constrain the dust characteristics.
\end{abstract}

Keywords. (ISM:) dust, extinction

\section{Introduction}

The absorption feature at $2175 \AA$ is widely observed in the ISM which implies that its carrier is made of commonly available material in the ISM. The width and the extinction peak of the feature show large variation with line-of-sight $( \pm 12 \%)$ indicating a large chemical dispersion in the Galaxy, however, the peak value always accurs at $2175 \AA$ (Fitzpatrick \& Massa 2007). From the observations of thermal emission from ISM dust in the 3-60 $\mu \mathrm{m}$ range, and the microwave emission from ISM, it is now recognized that the interstellar grain population can include a substantial amount of ultra-small grains with PAH composition. PAH has strong absorption in the 2000 to $2500 \AA$ region and the expected carrier of the $2175 \AA$ feature is a mixture of PAHs. Moreover, ultra-small grain population is needed to reproduce the Far-UV rise in the extinction curve. The observed variation in the width of the feature would result from differences in the PAH mix.

\section{TAUVEX mission and data resource}

TAUVEX has three equivalent $20 \mathrm{~cm}$ aperture UV telescopes with $\mathrm{f} / 8$ beam and an effective focal length of $1600 \mathrm{~mm}$ which will be put in its orbit by India in mid 2008. It will image large parts of the sky in the wavelength region between 1400 and $3200 \AA$ with a spatial resolution of about $6^{\prime \prime}$ to $10^{\prime \prime}$, depending on the wavelength. TAUVEX will be used to measure the fluxes at $1770 \AA, 2180 \AA, 2680 \AA$ and $2220 \AA$ using narrowband filters SF1, SF2, SF3 and NBF1 respectively. These observations will provide us with three points for the interstellar extinction in the UV domain, including at $2175 \AA$ bump. TAUVEX can detect un-reddened early B stars brighter than $m_{\mathrm{v}}=14(\mathrm{~S} / \mathrm{N} \sim 10)$ in one scan. Details on the mission can be found in http://tauvex.iiap.res.in.

The previous all sky survey in UV was performed by the TD1 satellite with a controlled scan of the whole sky. It measured the absolute UV flux distribution between $2740 \AA$ and $1350 \AA$ of unreddened early B stars with brightness upto $m_{\mathrm{v}}=10$, and are compiled 

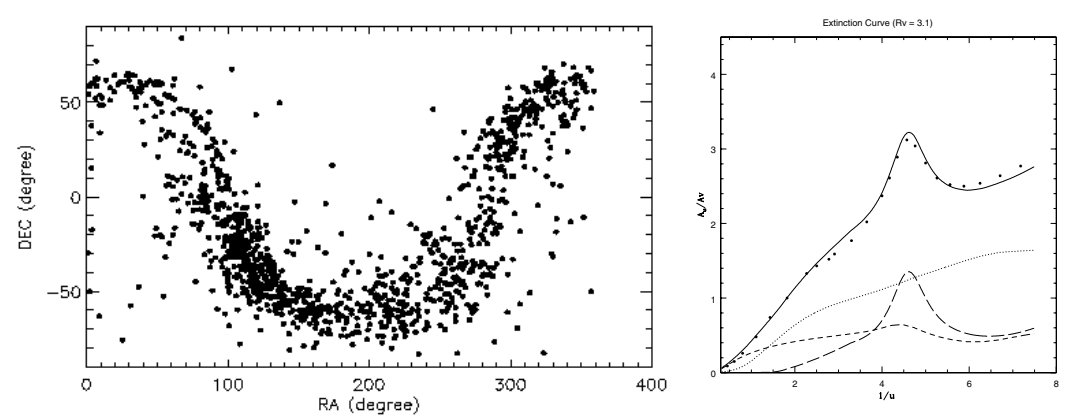

Figure 1. Left: The distribution of B0-B5 III-V stars obtained from the ASC catalog, Right: Extinction curve (solid line) produced using three component dust model: silicate (dotted), graphite (short dashed) and PAH (long dashed). The observations are shown in points.

Table 1. Values of the input model parameters

\begin{tabular}{l|c|c|c|c|c|c}
\hline & $\alpha$ & $\beta$ & $a_{t}(\mu)$ & $a_{c}(\mu)$ & $\mathrm{C}\left(10^{-11}\right)$ & $b_{c}\left(10^{-5}\right)$ \\
Silicate & -1.448 & -9.34 & 0.172 & 0.1 & 0.102 & - \\
Carbonaceous & -2.25 & -0.065 & 0.0075 & 0.606 & 9.94 & 2.0 \\
\hline
\end{tabular}

in the "Catalog of stellar UV fluxes" which can be used to obtain four more values for interstellar extinction at wavelengths 2740, 2365, 1965, and $1965 \AA$.

\section{Our Program}

In Figure 1, we present the distribution of B0-5 III-V stars obtained from All-Sky Compiled Catalog (ASC) of Kharchengo (2001) which will be used to select our program sources. From the photometric observations obtained from TAUVEX, TD1 and GALEX, we propose to investigate 1 ) the variation of $2175 \AA$ feature at different locations of the Galaxy and 2) to constrain the chemical composition of dust and size distribution at these locations. Taking the advantage of TAUVEX sensitivity, we expect to sample the distance upto $2 \mathrm{kpc}$ in the local ISM with a step interval of 50 to $100 \mathrm{pc}$ (Maheswar et al. 2007)

To model the extinction curve, we adopt the size distribution function introduced by Weingartner \& Draine (2001) for silicate and carbonaceous grains. This includes an ultrasmall grain population of size less than $100 \AA$ with PAH composition with the sum of two log-normal size distributions. These ultra small grains are in Rayleigh limit at $\lambda$ larger than $1000 \AA$ and hence constrains only their carbon abundances $\left(b_{c}\right)$. The size distribution of classical graphite and silicate grains of sizes larger than $0.1 \mu \mathrm{m}$ follows the power law. Carbonaceous grains having intermediate sizes between PAH and graphite have optical properties intermediate between those of PAH and graphite. Dielectric constants for the grains are taken from www.astro.princeton.edu/ draine and the extinction coefficients were calculated using BHMIE code.

Best fit model for the observed extinction curve is obtained by varying the carbon abundance $b_{c}$ transition sizes $a_{t, c}\left(a_{t, s}\right)$, upper cutoff size parameters $a_{c, g}\left(a_{c, s}\right.$, curvature parameters $\beta_{c}\left(\beta_{s}\right)$, powerlaw coefficients $\alpha_{c}\left(\alpha_{s}\right)$ for carbonaceous (silicate) grains. A model extinction curve produced for $R_{\mathrm{v}}=3.1$ from our program is shown in Figure 1 and the values of input parameters are listed in Table 1.

\section{References}

Fitzpatrick, E. L. \& Massa, D, 2007, ApJ, 663, 320

Kharchengo, N. V. 2001, ASC-2nd version, Kinematics and Physics of Celestial Bodies, 17, 400 Maheswar, G., Muthu, C., Sujatha, N. V., et al. 2007, Bull.Astron.Soc.India, 35, 233

Weingartner, J. C. \& Draine, B. T. 2001, ApJ, 548, 296 